Review Article

\title{
Pharmaceutical and Nutraceutical Potential Applications of Sargassum fulvellum
}

\author{
Jian Liu, ${ }^{1,2}$ Sibusiso Luthuli, ${ }^{1}$ Qifang Wu, ${ }^{1}$ Mingjiang Wu $\mathbb{D},{ }^{1}$ Jong-il Choi $\mathbb{D},{ }^{2}$ \\ and Haibin Tong ${ }^{1}{ }^{1}$ \\ ${ }^{1}$ College of Life and Environmental Science, Wenzhou University, Wenzhou 325035, China \\ ${ }^{2}$ Department of Biotechnology and Bioengineering, Chonnam National University, Gwangju 500-757, Republic of Korea \\ Correspondence should be addressed to Mingjiang Wu; wmj@wzu.edu.cn, Jong-il Choi; choiji01@chonnam.ac.kr, \\ and Haibin Tong; tonghaibin@gmail.com
}

Received 11 May 2020; Accepted 24 August 2020; Published 14 October 2020

Academic Editor: Ambikanandan Misra

Copyright ( 92020 Jian Liu et al. This is an open access article distributed under the Creative Commons Attribution License, which permits unrestricted use, distribution, and reproduction in any medium, provided the original work is properly cited.

Sargassum fulvellum is a brown seaweed of the Sargassaceae family which has been demonstrated to exhibit antipyretic, analgesic, antiedema, antimicrobial, antioxidant, antitumor, neuroprotective, anticoagulative, anti-inflammatory, and hepatoprotective activities. It has been widely used as a food additive and as a medicine in oriental medicine to treat lumps, dropsy, swelling, testicular pains, and urinary problems. S. fulvellum has been identified as a potential producer of a wide spectrum of natural compounds such as carotenoids, fucoidans, and phlorotannins, showing different biological activities in various industrial applications including pharmaceutical, nutraceutical, cosmeceutical, and functional food. However, the promising health effects associated with the extracts and compounds isolated from $S$. fulvellum have not been reviewed to date. The present review thus focuses on the biological activity of $S$. fulvellum as reported by previous publications, which include antioxidant, anticoagulant, anti-inflammatory, neuroprotective, immunomodulatory, antidiabetic, and anticancer effects. Thus, this review might serve to increase the utilization of this invaluable natural source as a potential component in pharmaceutical and nutraceutical applications.

\section{Introduction}

Seaweeds are used both as medicinal ingredients and as healthy foods in East-Asian countries such as China, Korea, and Japan [1]. Over the last years, seaweeds have been garnering considerable interest as sources of valuable functional metabolites [2]. The interest toward these biologically active components arises from their increasing potential as functional components in pharmaceutical, food, and cosmetic industries $[3,4]$.

Sargassum fulvellum (Turner) C. Agardh belongs to the brown seaweed (Phaeophyceae) order of the Sargassaceae family and is widely distributed throughout the Korean, Chinese, and Japanese coasts. It is also known as "Wu Lei Ma Wei Zao" (无肋馬尾藻) in Chinese, “Hondawara” (馬尾 藻) in Japanese, and "Mojaban" (모자반) in Korean. This seaweed is regarded as an edible seaweed, and its dry material contains high contents of carbohydrates (30-60\%), such as cellulose, fucoidan, laminaran, alginic acid, protein (15.8\%), ash $(27.5 \%)$, and relatively low amounts of fat $(5 \%)[5,6]$. People living in Jeju Island (Republic of Korea) use this seaweed to prepare salads, soups (Mom-Guk), or side dishes [7-9]. In addition to its nutritional value, traditional medicine recommends the use of $S$. fulvellum to treat diseases such as a lumps, swelling, testicular pains, and urinary tract infections [10-12]. Previous studies have reported that $S$. fulvellum contains many bioactive molecules, such as phlorotannins, grasshopper ketone, fucoidan, and polysaccharides. S. fulvellum extracts or isolated pure components have been studied for years for analyzing their diverse pharmacological effects such as antioxidant, anticancer, antiinflammatory, antibacterial, and anticoagulant activities [11, 13-15].

Since 2000, more than 40 papers have been published on the bioactive properties of $S$. fulvellum. However, there is still insufficient systemic data on its chemical constituents and 
pharmacological effects. This paper summarizes the research conducted over the past decades, on the chemical components of S. fulvellum and their biological activities. Nowadays, the therapeutic effects of this seaweed are scientifically credible and have been partially explained by in vivo and in vitro assays. Hence, our review summarized the literature on the biological characterization and pharmacological bioactivities of S. fulvellum, with an emphasis on its functional properties, which could provide a comprehensive insight into the chemical structures and biological activities of its active compounds and could also provide information that would lead to the development of more applications based on $S$. fulvellum.

\section{Search Strategy, Data Collection, Evaluation, and Screening}

In the current review, the relevant information on S. fulvellum was gathered from worldwide accepted scientific and academic databases via an electronic search such as Google Scholar, Web of Science, ScienceDirect, PubMed, Wiley Online Library, and Scopus. The following search terms were used as keywords and Boolean operator: "Sargassum fulvellum" + "extracts/compounds" or "polysaccharide" or "fucoidan" or "characterization" or "purification"; "Sargassum fulvellum" + "anticancer/antitumor" or "antioxidant" or "anti-inflammatory" or "immunomodulatory" or "anticoagulative" or "neuroprotective" or "hepatoprotective". The review accessed and summarized the literature published until March 2020. No restrictions to any specific languages were made. All studies that met title and abstract criteria were selected for full-text review. A total of 84 research and review articles were reviewed for all mentioned databases; and only 46 potential research and review articles were screened out containing the maximum relevant information under the umbrella of different keywords. Afterwards, the authors carefully studied the selected articles in terms of content to write this review.

\section{Important Biologically Active Components of S. fulvellum}

Marine organisms are rich sources of structurally diverse bioactive compounds, and their importance as sources of novel bioactive substances is growing rapidly [16]. S. fulvellum is a source of many health-promoting components, mainly phlorotannins, fucoxanthin, and polysaccharides. Surprisingly, phlorotannins have been found only in marine brown algae, which protect them from natural enemies by acting as herbivore deterrents, digestive inhibitors, ultraviolet (UV) screens, and antibacterial agents [17, 18]. Phlorotannins are structurally less complex than terrestrial tannins, as they comprise chains of 1,3,5-trihydroxybenzene formed via the acetate-malonate pathway $[19,20]$. The phlorotannins can be categorised into three primary types according to the criteria of interphloroglucinol linkages: (i) fucols, (ii) phlorethols, and (iii) fucophlorethols (Figure 1) [21, 22]. Fucoxanthin (Figure 2(a)) is a well-known xanthophyll pigment, responsible for the brown color of seaweeds; it accounts for around $10 \%$ of the total natural seaweed carotenoid content [23]. Interesting bioactive properties are associated with fucoxanthin, which is a popular functional ingredient in food, cosmeceutical, and nutraceutical industries $[24,25]$. However, the bioavailability of marine algaederived bioactive small molecules like fucoxanthin or phlorotannins has not yet been sufficiently researched, especially in S. fulvellum. In a recent study [6], the grasshopper ketone (GK) (Figure 2(b)) was isolated and purified from the $n$-hexane fraction from $S$. fulvellum. Its structure was elucidated on the basis of spectral and chemical evidence and demonstrated that GK has anti-inflammatory activity and contributes to the treatment of inflammatory diseases. Ina et al. previously isolated an analog to chlorophyll-related compounds, pheophytin A (Figure 2(c)), from S. fulvellum and demonstrated that it is a neurodifferentiation compound [26]. According to $\mathrm{Wu}$ et al., two bioactive products identified as 1-O-palmitoyl-2-O-oleoyl-3-O-( $\alpha$-D-glucopyranosyl)-lycerol and 1-Omyristoyl-2-O-oleoyl-3-O-( $\alpha$-D-glucopyranosyl)-glycerol (Figure 2(d)) obtained from S. fulvellum showed fibrinolytic activity in the reaction system of single-chain urokinasetype plasminogen activator (pro-u-PA) and plasminogen [27].

S. fulvellum is known to produce different polysaccharides, like fucoidans (Figure 2(e)), alginates (Figure 2(f)), and laminarans (Figure 2(g)). Laminarans and fucoidans are the main water-soluble polysaccharides in brown algae, whereas high-molecular mass alginic acids represent alkalisoluble polysaccharides. Alginic acid has two basic components, $\alpha$-1,4-linked L-guluronic acid and $\beta$-1,4-linked Dmannuronic acid (both of which are hexuronic acids), arranged in homopolymeric blocks separated by regions of alternating sequences of the two monomers [28]. Fucoidans are a class of sulfated polysaccharides commonly present in the extracts of brown seaweeds [29]. Fucoidan is largely composed of ${ }_{L}$-fucose and sulfate groups. It is used worldwide, especially by the food and pharmaceutical industries because of its diverse bioactive effects like anticoagulant, antioxidant, anticancer, anti-inflammatory, and immune-modulatory activities. Fucoidan is a homofucose polysaccharide usually classified into the following two types: the first (I) is composed of repeated $(1 \rightarrow 3){ }_{L}$-fucopyranose units, while the other type (II) alternates repeated $(1 \rightarrow 3)$ - and $(1 \rightarrow 4){ }_{\mathrm{L}^{-}}{ }^{-}$ fucopyranose units. Laminaran is a major storage polysaccharide present in brown seaweeds [30]. It represents $35 \%$ of the dry weight of seaweeds. Laminaran consists of glucose monomers joined together mainly by a $(1,3)-\beta$-D-glucan backbone with $\beta(1,6)$ glycosidic bonds. In recent years, brown seaweeds have become important sources of natural compounds [31-33], many of which have been proven to possess bioactive effects $[19,34]$. Therefore, the need to isolate novel bioactive compounds from edible seaweeds has increased recently.

\section{Biological Activities of S. fulvellum}

4.1. Antioxidant Activity. Oxidative stress has been associated with a number of lifestyle-related diseases, such as aging, arthritis, atherosclerosis, emphysema, cancer, and diabetes 


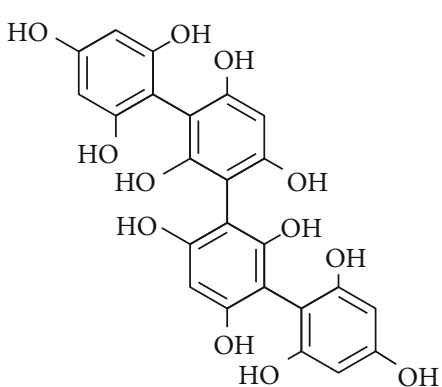

(a)<smiles>Oc1cc(O)cc(Oc2c(O)cc(Oc3c(O)cc(O)c(Oc4cc(O)cc(O)c4O)c3O)cc2O)c1</smiles>

(b)<smiles>Oc1cc(O)c(Oc2cc(O)c(-c3c(O)cc(O)cc3O)c(O)c2)c(O)c1</smiles>

(c)

Figure 1: Example structures of the primary types of phlorotannins: fucols (a); phlorethols (b); fucophlorethols (c).

[35]. Marine seaweeds have been considered one of the main sources of natural antioxidants. Antioxidant properties in vivo and in vitro were tested in different ways, including free radical scavenging, lipid peroxidation inhibition, and the enhancement of superoxide dismutase (SOD), catalase (CAT), and glutathione peroxidase (GSH-Px) activities [36]. An antioxidant evaluation was performed on fucoidan (named SFSP, molecular weight $(\mathrm{MW})=529 \mathrm{kDa}$ ) extracted from S. fulvellum harvested from Jeju City. SFSP was selected because it exhibited more potent activity than the commercially available antioxidants, butylated hydroxyanisole and $\alpha$-tocopherol. Results showed a dose-dependent hydrogen peroxide scavenging activity in the V79-4 cell line and a promising $\alpha, \alpha$-diphenyl- $\beta$-picrylhydrazyl (DPPH) free radical scavenging activity when compared to the commercial fucoidans (Fucus vesiculosus and Undaria pinnatifida) [7]. These fucoidans contained a significant amount of phenolic compounds, which suggested that the antioxidant activities of fucoidan could be attributed to the combined effect of polysaccharides and phenolic compounds. Interestingly, a study on the antioxidant activity of S. fulvellum methanolic extract (named SFME) demonstrated that it can strongly scavenge reactive oxygen species (ROS) such as superoxide anions, hydroxyl radicals, hydrogen peroxide, and DPPH free radicals. In addition, SFME also showed higher polyphenolic content $(7.56 \mathrm{mg} / \mathrm{g})$, which was correlated with superoxide anion and DPPH free radical scavenging abilities [37]. These results indicate that further investigations are needed to identify and purify the responsible antioxidative components. Wang et al. [36] investigated the antioxidant properties of polysaccharides in S. fulvellum extracts prepared using Celluclast (SFC) and found that SFCs scavenged $\mathrm{DPPH}$, alkyl, and hydroxyl radicals, especially the alkyl radical $\left(\mathrm{IC}_{50}=0.45 \pm 0.11 \mathrm{mg} / \mathrm{mL}\right)$. Additionally, SFCs significantly reduced intracellular ROS levels and improved the viability of 2,2-azobis(2-amidinopropane) hydrochloride-
(AAPH-) induced Vero cells in a dose-dependent manner. To date, the relationships between the antioxidant activity, structural features, and chemical structure of various polysaccharides are still unclear. Fortunately, various reports have indicated that the antioxidant activities of polysaccharides may be correlated with intrinsic viscosity and MW $[38,39]$, as well as monosaccharide composition, chemical structures, uronic acid content, and chain conformations [40]. Future studies also need to investigate the mechanisms underlying $S$. fulvellum antioxidant activity. They should also aim to improve antioxidant inhibition assays, which will be helpful for understanding the relationship between the chemical structures and antioxidant properties. The summarized antioxidant activities of the biologically active components of S. fulvellum provide a comprehensive evaluation of its value as a natural antioxidant source (Table 1).

4.2. Neuroprotective Activity. With the increase in life expectancy, normal brain aging and neurological disorders due to pathological degeneration of neurons are a growing concern for the elderly. The pathogenesis of neurological disorders tends to be associated with region-specific neuronal degeneration and atrophy, which is primarily caused by the reduced level of neurotrophic factors [41, 42]. The development of effective neuroprotective agents is important for the treatment of both acute and chronic brain disorders, such as traumatic brain injury, stroke, and Alzheimer's and Huntington's diseases $[43,44]$. Seaweeds are potential sources for such agents [45]. So far, three compounds with neuritogenic activity have been identified from the genus Sargassum. Sargaquinoic acid and sargachromenol from Sargassum macrocarpum were found to promote neurite outgrowth in rat pheochromocytoma (PC12) cells in the presence of nerve growth factor at low concentrations $[22,46]$. Another neurodifferentiation compound, pheophytin A (Figure 1(c)), identified and characterized from S. fulvellum, was shown to have a similar 


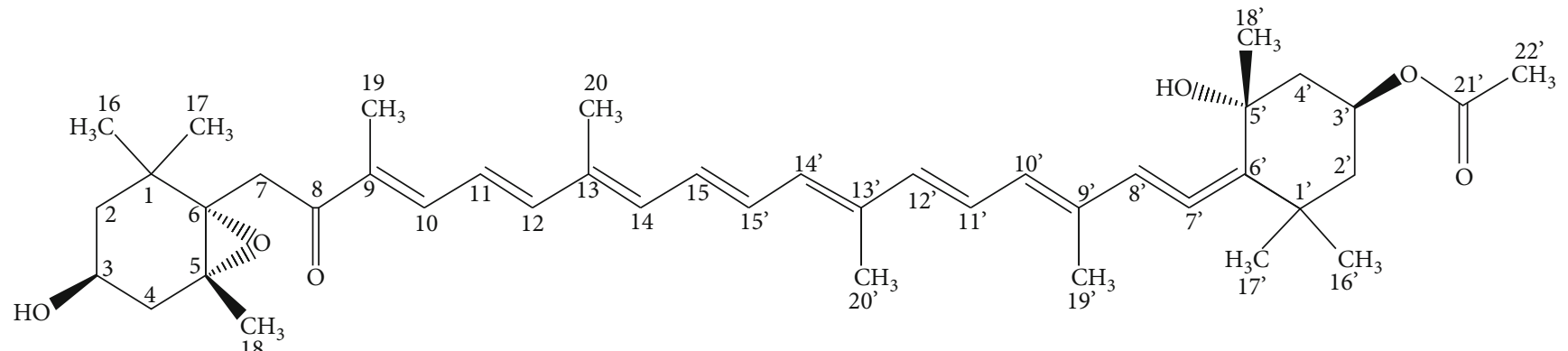

(a) All-trans-fucoxanthin

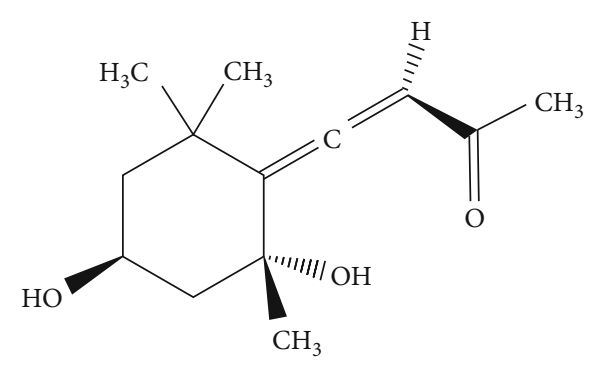

(b) Grasshopper ketone

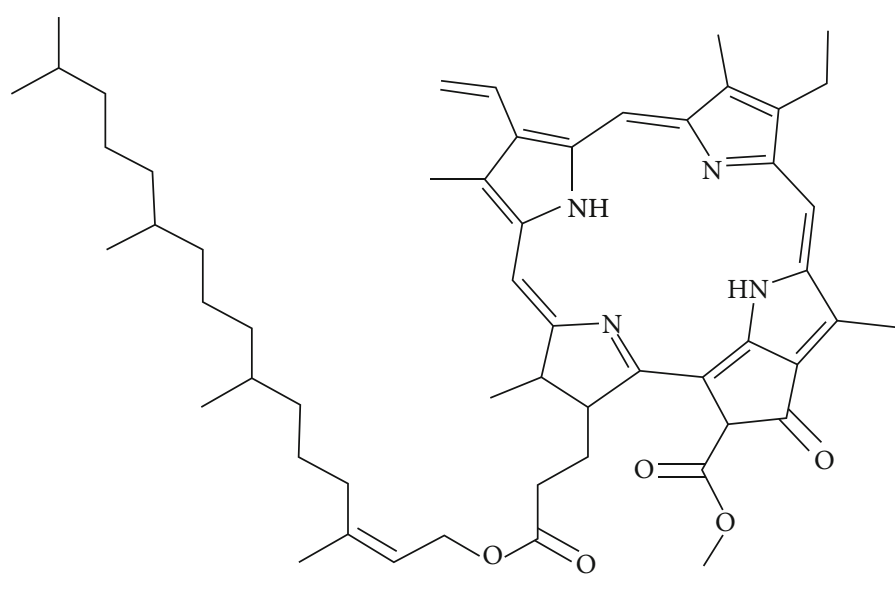

(c) Pheophytin A<smiles>[R10]OC(CO)CO[C@H]1C[C@@H](CO)[C@@H](O)[C@H](O)[C@H]1O</smiles>

1: R1 = palmitoyl R2 = oleoyl

2: $\mathrm{R} 1=$ myristoyl $\mathrm{R} 2=$ oleoyl

(d) Glucopyranosyl diacylglycerol
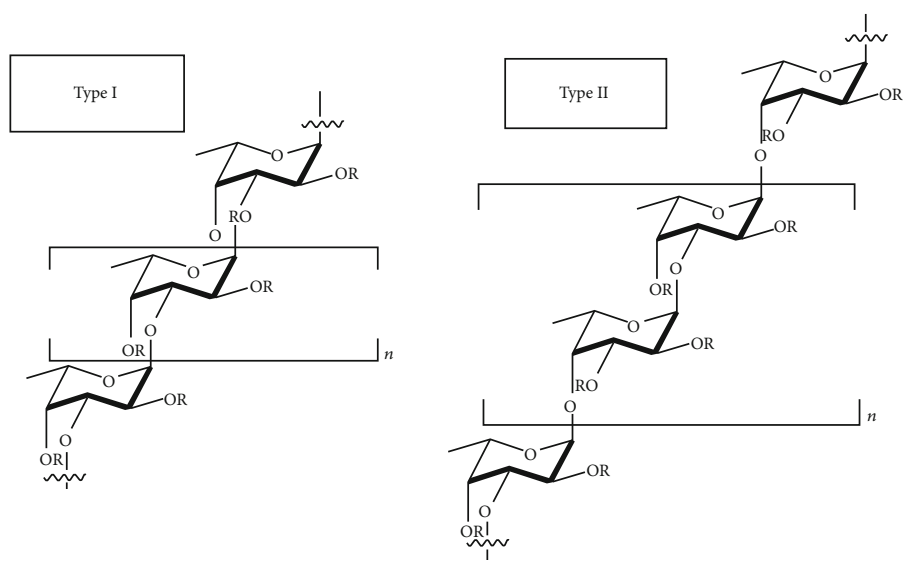

(e) Fucoidan

Figure 2: Continued. 


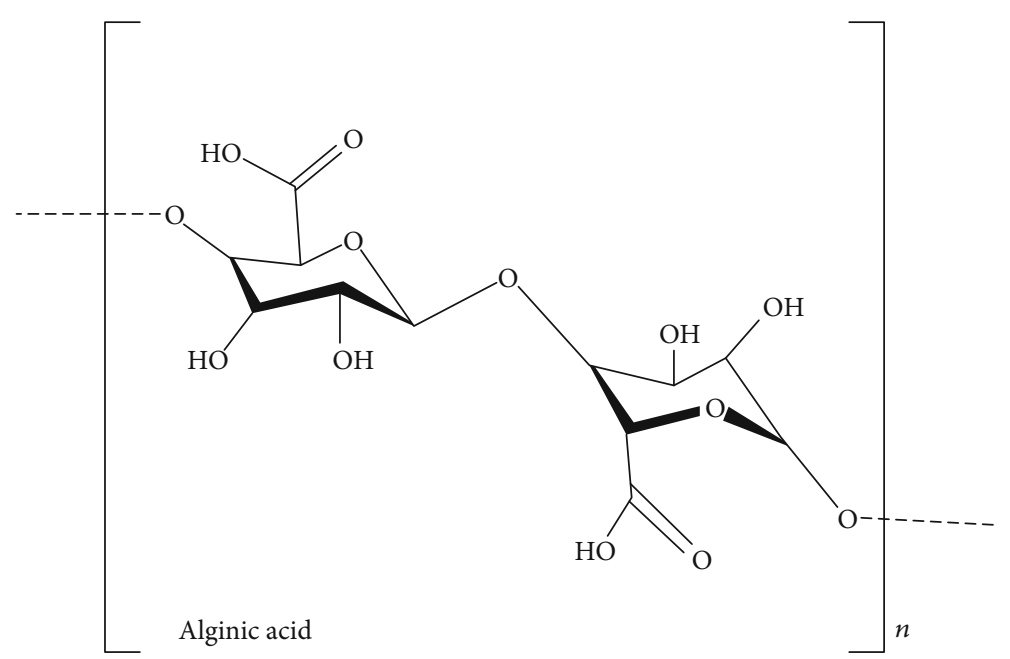

(f) Alginic acid

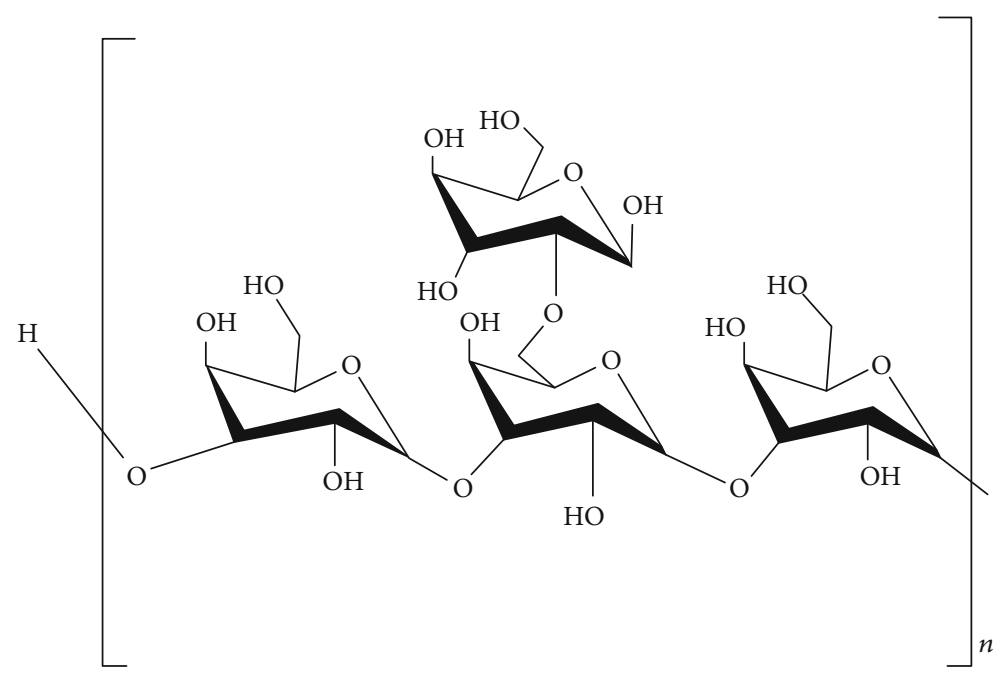

(g) Laminaran

Figure 2: Structures of bioactive compounds isolated from S. fulvellum.

neuritogenic activity in PC12 cells [26]. This study suggested that pheophytin A at a concentration of $3.9 \mu \mathrm{g} / \mathrm{mL}$ synergizes with nerve growth factor (NGF) to promote neurite outgrowth in rat pheochromocytoma PC12 cells via mitogenactivated protein kinase signaling. Moreover, in the followup study, the same group investigated the effects of the pheophytin $\mathrm{A}$ analog vitamin $\mathrm{B}_{12}$ on PC12 cell differentiation. Vitamin $\mathrm{B}_{12}$ demonstrated neurite outgrowth-promoting activity in PC12 cells, and in a manner similar to NGF, it stimulates the mitogen-activated protein kinase (MAPK) signaling pathway via the activation of extracellular signalregulated kinase (ERK) [47]. However, it should be noted that PC12 cells are neurosecretory cells that originate from the peripheral nervous system and that they do not form neurites in vivo. Another point to note is that these compounds do not have the potential to initiate neuritogenesis by themselves; instead, they can enhance the neurite outgrowth of PC12 cells in the presence of low concentrations of nerve growth factor, but further efforts are required to evaluate the potential neurotrophic effects of S. fulvellum on neurons from the central nervous system (CNS). Hannan et al. [45] investigated the ethanol extracts of 22 seaweed species, including Gelidium amansii, Undaria pinnatifida, and $S$. fulvellum for their ability to protect mature neurons from atrophy in neurodegenerative diseases. In this preliminary screening experiment, the ethanol extracts of S. fulvellum (SFE) showed neurite outgrowth-promoting ability, thus helping the development of rat hippocampal neurons. In another study, the authors extended the previously reported neurite outgrowth-promoting activity of S. fulvellum in the PC12 cells to the CNS neurons and further proved its efficacy with respect to the formation of neural networks by enhancing synaptogenesis. Treatment of rat hippocampal neurons with SFE $(5 \mu \mathrm{g} / \mathrm{mL})$ resulted in a significant enhancement of desirable effects, such as neuronal maturation in the early stage of development, cytoarchitecture complexity, and synaptogenesis in the later maturation stage. In addition to its effect on neurite development, SFE significantly increased the number of postsynaptic density-95 and synaptic vesicle 2 puncta and synapses (about 35\%, 67\%, and 125\%, 
TABLe 1: Summary of the biological activities of the brown seaweed S. fulvellum.

\begin{tabular}{|c|c|c|c|}
\hline Therapeutic activity & Extract/compound & Pathway mode/effect & References \\
\hline \multirow{3}{*}{ Antioxidant } & Fucoidan & $\begin{array}{l}\mathrm{DPPH}, \mathrm{HO}, \mathrm{NO} \text { radical scavenging } \\
\text { Hydrogen peroxide scavenging }\end{array}$ & {$[7]$} \\
\hline & Enzymatic extract & DPPH radical scavenging & {$[76]$} \\
\hline & $\begin{array}{l}\text { Enzyme-assistant } \\
\text { extracts }\end{array}$ & $\begin{array}{l}\text { DPPH, alkyl, and hydroxyl radical scavenging } \\
\text { Reduced intracellular ROS levels }\end{array}$ & {$[36]$} \\
\hline \multirow[b]{2}{*}{ Neuroprotective } & Pheophytin A & Activation of a MAPK signaling pathway & {$[26]$} \\
\hline & Ethanol extract & $\begin{array}{l}\text { Promoted the initial neuronal differentiation and increased the indices of } \\
\text { axonal and dendritic development }\end{array}$ & {$[45]$} \\
\hline \multirow{12}{*}{ Anti-inflammatory } & Ethanol extract & Suppressed production of NO, IL- 6 , TNF- $\alpha$, and IL- $1 \beta$ & [79] \\
\hline & Water extract & Suppressed the NO and TNF- $\alpha$ secretion & {$[55]$} \\
\hline & $\begin{array}{c}\text { Seaweed } \\
\text { supplementation }\end{array}$ & Reduced circulation of IL- $1 \beta$ and IL- 6 & {$[80]$} \\
\hline & Grasshopper ketone & Inhibiting MAPKs (ERK, JNK, and p38) and NF- $\kappa$ B p65 phosphorylation & {$[6]$} \\
\hline & Aqueous extract & Inhibited NO production & {$[56]$} \\
\hline & Water extract & Inhibited NO production & {$[53]$} \\
\hline & Hexane fraction & $\begin{array}{l}\text { Downregulation of NF- } \kappa \mathrm{B} \text { activity via the inhibition of MAPKs and Akt } \\
\text { phosphorylation }\end{array}$ & {$[10]$} \\
\hline & Grasshopper ketone & Regulatory effects of IL-4, IL-5, IL-10, IL-13, IFN- $\gamma$, and TNF- $\alpha$ & {$[58]$} \\
\hline & Hot-water extract & $\begin{array}{l}\text { Inhibited the expression levels of IL- } 1 \beta \text {, IL- } 4 \text {, IL-5, IL-6, IL-8, and IL-13 via } \\
\text { NF- } \kappa \text { B } \\
\text { Activated ERK/P38 }\end{array}$ & {$[48]$} \\
\hline & Ethanolic extract & Suppressed NF- $\kappa$ B activation & {$[57]$} \\
\hline & Ethanolic extract & $\begin{array}{l}\text { Inhibited the expression of proinflammatory proteins such as COX-2, TNF- } \alpha \text {, } \\
\text { and iNOS }\end{array}$ & {$[62]$} \\
\hline & Water extract & Modulation of IL-4 and IFN- $\gamma$ & [81] \\
\hline \multirow{2}{*}{ Anticoagulative } & Enzymatic extract & Inhibited the serine proteases II, X, and VII & {$[68]$} \\
\hline & Fucoidan & Inhibited both intrinsic and extrinsic blood coagulation pathways & {$[15]$} \\
\hline \multirow{3}{*}{ Antidiabetic } & $\begin{array}{c}\text { Seaweed } \\
\text { supplementation }\end{array}$ & Reduced insulin resistance & {$[80]$} \\
\hline & Enzymatic extract & $\alpha$-Glucosidase inhibitory & {$[76]$} \\
\hline & Ethanolic extract & $\alpha$-Glucosidase inhibitory & {$[75]$} \\
\hline \multirow{3}{*}{ Immunomodulatory } & Polysaccharides & Via activation of NF- $\kappa$ B and MAPK signaling and the Th1 immune response & {$[13]$} \\
\hline & $\begin{array}{l}\text { Polysaccharides and } \\
\text { ethanol extract }\end{array}$ & Increased splenocyte proliferation and production of Th1-type cytokines & {$[54]$} \\
\hline & Grasshopper ketone & Inhibition of $\mathrm{T}$ cell activation & {$[58]$} \\
\hline \multirow{2}{*}{ Hepatoprotective } & $\begin{array}{c}\text { Seaweed } \\
\text { supplementation }\end{array}$ & Increase of GSH level in cecal contents & {$[73]$} \\
\hline & Fucoidan & $\begin{array}{l}\text { Through intestinal bacteria fermentation, but further study is necessary to } \\
\text { completely clarify }\end{array}$ & {$[72]$} \\
\hline \multirow{5}{*}{ Anticancer } & Hot-water extract & Upregulation of p53 & {$[77]$} \\
\hline & Sodium alginate & Inhibited uptake of ${ }^{3} \mathrm{H}$-thymidine & {$[82]$} \\
\hline & Polysaccharide & Inhibited the growth of subcutaneous sarcoma- 180 & {$[14]$} \\
\hline & Hot-water extract & Inhibited the growth of subcutaneous sarcoma- 180 & {$[83]$} \\
\hline & Organic solvent extract & High cytotoxicity to HepG2, HT-29, and HeLa & [77] \\
\hline Fibrinolytic & $\begin{array}{c}\text { Glucopyranosyl } \\
\text { diacylglycerols }\end{array}$ & Enhanced reciprocal activation of pro-u-PA and plasminogen & {$[27]$} \\
\hline Antimicrobial & Ethanol extract & Antibacterial & {$[74]$} \\
\hline Antithrombotic & Fucoidan & Prevented P-selectin binding & {$[69]$} \\
\hline
\end{tabular}


respectively, with respect to that in the control) [12]. Notably, small molecules isolated from S. fulvellum showed higher protective effect against neurite damage in cases of excessive neuronal cell loss, indicating that $S$. fulvellum can promote neuronal maturation and synaptogenesis, and support neuronal survival. Thus, $S$. fulvellum could have potential therapeutic value for brain aging as well as for neurodegenerative diseases by favoring the reconstruction of the partially damaged neuronal network.

4.3. Immunoinflammatory Activity. Seaweeds produce bioactive compounds, including fucoidans, fucosterol, and phlorotannins, which are responsible for regulating immunesignaling and anti-inflammatory activity [48-52]. S. fulvellum has been shown to possess immunomodulatory properties that are potentially applicable for stimulating the immune response or controlling immune cell activity for mitigating associated negative effects such as inflammation [53]. These marine sources of medicinal compounds may also affect multiple targets in the immune and inflammatory systems that are important for disease progression. The immunomodulatory activities of a S. fulvellum polysaccharide (SFP) have been investigated previously in RAW 264.7 macrophages and mouse splenocytes in vitro by Sung et al. [13]. It was found that SFP induced macrophage activation by increasing the expression of CD80 and CD86 molecules, and it resulted in the production of markedly higher levels of proinflammatory cytokines (tumor necrosis factor(TNF-) $\alpha$, interleukin- (IL-) 6 , and IL-12p70) through the activation of nuclear factor kappa B (NF- $\kappa \mathrm{B})$, MAPK signaling, and the T helper 1 (Th1) immune response. Similarly, Byun [54] compared the immunomodulatory effects of SFP with those of SFE in macrophages and murine splenocytes and confirmed that the immunomodulation may be dependent on polysaccharide extracts. This study demonstrated that SFP significantly increases nitric oxide (NO) and cytokine production (TNF- $\alpha$, IL-6, and IL- $1 \beta$ ), whereas SFE does not cause increased $\mathrm{NO}$ and cytokine production. In the case of splenocytes, SFP increased splenocyte proliferation and Th1 type cytokine (IL-2 and interferon- (IFN-) $\gamma$ ) production to a greater extent than SFE. The above results suggested that the immunomodulatory activity of SFP was mainly mediated via the regulation of Th1-related cytokine expression. The time-effect relation of the cytokine response also indicated that macrophages and natural killer cells involved in nonspecific immunity were primarily activated and that helper $\mathrm{T}$ cells were secondarily affected in response to SFP treatment.

Direct stimulation of immune cells by $S$. fulvellum extracts results in the production of NO through the induction of inducible nitric oxide synthase (iNOS) and in the generation of a proinflammatory cytokine/chemokine profile. Depending on the situation, the interaction of S. fulvellum extracts with other effectors may result in reduced inflammation. For example, NO inhibition was highly increased in lipopolysaccharide- (LPS-) treated macrophages in the presence of $S$. fulvellum aqueous extracts. Studies also showed that S. fulvellum extracts increased RAW 264.7 macrophage proliferation by more than $80 \%$ [53]. Jeong et al. [55] confirmed that NO and TNF- $\alpha$ secretion were significantly inhibited when LPS-stimulated RAW 264.7 cells were treated with S. fulvellum water extracts (SFWE). Moreover, SFWE inhibited the expression of IL- 6 and IL- $1 \beta$ in a dosedependent manner. In particular, IL-6 inhibition activity was $>50 \%$ at a concentration of $1 \%$ SFWE. Jaswir et al. [56] showed that $S$. fulvellum ethanol extract (SFEE) inhibited not only the production of NO and proinflammatory cytokines (IL-6, IL- $1 \beta$, and TNF- $\alpha$ ) but also the expression of iNOS and cyclooxygenase 2 in LPS-stimulated RAW 264.7 cells without affecting their viability. SFEE also suppressed the expression of NF- $\kappa \mathrm{B}$, suggesting that SFEE could affect the expression of inflammation-related cytokines and proteins through the regulation of NF- $\kappa \mathrm{B}$. Furthermore, the formation of ear edema was $40 \%$ lower in mice treated with the highest dose $(250 \mathrm{mg} / \mathrm{kg})$ of SFEE than that in the control mice [57].

There is cumulative evidence to suggest that $S$. fulvellum extracts have anti-inflammation potential that modulate pro/anti-inflammatory cytokine secretion profiles. Gwon et al. [10] investigated the inhibitory effect of hexane extract from S. fulvellum on the LPS-stimulated RAW 264.7 cells and phorbol 12-myristate 13-acetate- (PMA-) induced mouseear edema. The hexane fraction of S. fulvellum (HFS) inhibits the production of proinflammatory mediators and cytokines. Moreover, the anti-inflammatory effects of HFS on LPStreated cells are associated with the inactivation of the NF$\kappa \mathrm{B}$ pathway implicated with the suppression of $\mathrm{NF}-\kappa \mathrm{B}$ through the inhibition of MAPKs and Akt (cellular homolog of murine thymoma virus akt8 oncogene) phosphorylation. Verification of HFS anti-inflammatory activity and relative mechanism at the cellular and molecular levels will be beneficial for its application in therapeutic agents for treating inflammation-mediated diseases. Recently, the grasshopper ketone (GK) (Figure 1(b)) extracted from S. fulvellum showed anti-inflammatory activities in LPS-induced RAW 264.7 murine macrophage cell line. The production of proinflammatory cytokines (IL-6, IL- $1 \beta$, and TNF- $\alpha$ ) was significantly reduced in $0.1-100 \mu \mathrm{g} / \mathrm{mL}$ dose ranges of $\mathrm{GK}$ treatment. Furthermore, the dose-dependent and significant inhibition of iNOS and cyclooxygenase-2 (COX-2) protein expression was confirmed. The GK exerts its antiinflammatory effect by inhibiting MAPKs (ERK, c-Jun-Nterminal kinase (JNK), and p 38 kinase) and NF- $\kappa$ B p 65 phosphorylation [6]. In another study, GK isolated from SFEE had an inhibitory effect on atopic dermatitis (AD) on 2,4dinitrochlorobenzene- (DNCB-) induced AD-like skin lesions in $\mathrm{BALB} / \mathrm{c}$ mice. In this study, SFEE inhibited the development of $\mathrm{AD}$-like skin lesions and decreases serum IgE and cytokine levels (IL-4, IL-5, IL-10, IL-13, IFN- $\gamma$, and TNF- $\alpha$ ), while the GK suppressed the expression of IFN- $\gamma$ and IL-4 in mouse splenocytes [58]. Overall, this study demonstrated that the anti-inflammatory properties of GK on macrophages were achieved by inhibiting the NF- $\kappa \mathrm{B}$ and MAPK pathways, which are associated with the attenuation of cytokine secretion [6].

As is well known, UV radiation is an important etiologic factor for inflammatory skin damages, oxidative stress, DNA damage, cellular and tissue injuries, cell death, skin cancer, and premature skin aging [59-61]. In a study performed by 
Lee et al. [62], the experimental HaCaT keratinocytes and $\mathrm{BALB} / \mathrm{c}$ mice were treated with the ethyl acetate fraction of S. fulvellum ethanol extract (SFE-EtOAc); it was found that SFE-EtOAc could be an effective anti-inflammatory agent protecting against UVB irradiation-induced skin damage. The results showed that SFE-EtOAc effectively inhibited UVB-induced cytotoxicity and the production of proinflammatory proteins or mediators including $\mathrm{COX}-2, \mathrm{TNF}-\alpha$, iNOS, prostaglandin (PG) E2), and $\mathrm{NO}$, both in in vitro $\mathrm{HaCaT}$ human keratinocytes and in in vivo BALB/c mice.

These and other studies report of bioactive compounds derived from S. fulvellum with immunomodulatory and anti-inflammatory properties. However, the effect of $S$. fulvellum on immunoinflammatory activities and its underlying molecular mechanisms have not been studied and remain largely unresolved. Further studies are required to determine the anti-inflammatory potential of S. fulvellum extracts and their application in food and pharmaceutical products. These extracts should be further purified and characterized to possibly identify the relationship between their antiinflammatory role and chemical structure, which would provide a novel insight into the application of S. fulvellum in the development of alternative anti-inflammatory drugs.

4.4. Anticoagulant and Antithrombotic Effects. Anticoagulants inhibit or decrease the ability of blood to clot or coagulate and therefore help preventing the formation of harmful clots in blood vessels [63]. Heparin is a highly sulfated polysaccharide present in mammalian tissues and a widely used anticoagulant for the treatment and prevention of thrombotic diseases [64]. Alternative drugs for heparin are in high demand, due to its adverse effects, such as occasional lifethreatening bleeding and thrombocytopenia. Thus, the disadvantages associated with heparin motivate further research on novel substances with anticoagulant properties [35]. Thus, anticoagulant and antithrombotic activities are the most studied properties of sulfated polysaccharides from seaweeds [65-67].

The compounds and extracts of $S$. fulvellum also possess anticoagulant and antithrombotic effects, and it was thought that sulfated polysaccharides were responsible for this [68]. De Zoysa et al. [15] found that the anticoagulant activity of fermented S. fulvellum sulfated polysaccharides was closely related to the inhibition of both intrinsic and extrinsic pathways of coagulation, but not to the inhibition of thrombin activity or fibrin polymerization. Freeze-dried S. fulvellum was fermented in an incubator for 10 weeks at $25^{\circ} \mathrm{C}$ to convert seaweed macromolecules into anticoagulant sulfated polysaccharides (ASP). The anticoagulant activity of ASP was measured using activated partial thromboplastin (APTT), thrombin time (TT), and prothrombin (PT) clotting time assays. The results suggested that both ASP and heparin showed a relative clotting factor of 27.47 at the concentrations of 180 and $60 \mu \mathrm{g} / \mathrm{mL}$, respectively. Chemical characterization further showed that $S$. fulvellum ASP had a considerable amount of negatively charged sulfate, which may be able to interact with coagulation factors and thus initiate the coagulation inhibition [15]. In another study, Jo and Choi [69] investigated the P-selectin binding and the anti- thrombotic and hemolytic activity of the depolymerized fucoidans extracted from $S$. fulvellum. The fucoidans degraded by ultrasound (US) or electron beam (EB) irradiation in the presence of hydrogen peroxide aqueous solution and the low molecular weight fucoidans (LMWFs) prevented $\mathrm{P}$-selectin binding to Sialyl Lewis $\mathrm{X}$ with an $\mathrm{IC}_{50}$ (inhibitory concentration 50) of $20 \mathrm{nM}$ as compared to heparin $(400 \mathrm{nM})$ and dextran sulfate $(25,000 \mathrm{nM})$. This means that LMWFs in this study were much more effective in antithrombotic activity than heparin and dextran sulfate. Notably, the LMWFs showed no hemolytic activity at concentrations of up to $950 \mu \mathrm{g} / \mathrm{mL}$. Thus, it might be justified to state that LMWF is safer than heparin and its constituents in clinical use. These results showed that $S$. fulvellum sulfated polysaccharide (fucoidan) has high blood anticoagulation activity and significantly prolongs the time preceding thrombosis and shortens thrombosis duration.

4.5. Hepatoprotective Activity. Despite the huge advancements in modern medicine, drugs able to effectively stimulate hepatic function offer complete protection to the liver, or aid in the regeneration of hepatic cells is yet undiscovered. Additionally, some drugs also induce adverse or side effects. Thus, it is necessary to develop alternative pharmaceuticals for the treatment of hepatic diseases, based on more effective and less toxic agents $[70,71]$. Kawano et al. [72] reported that diets containing edible seaweeds ( $S$. fulvellum) prevented liver toxicity induced by $\mathrm{D}$-galactosamine in rats. It also normalized the elevated levels of serum alanine transferase (ALT) and those of GSH in the cecum. However, they have not clarified the components or factors in S. fulvellum able to repress D-GalN-hepatopathy [73]. In a following study, the authors suggested that the protective effect of the three kinds of S. fulvellum against D-GalN-hepatopathy could be partly attributed to fucoidan. This study proposed one plausible mechanism, i.e., the marked increase of GSH levels in cecal contents as a result of repeated intake of S. fulvellum could promote the D-GalN-hepatopathy defense mechanism by influencing bacterial translocation to the intestine. The obtained results demonstrate the hepatoprotective activity of $S$. fulvellum fucoidan and provide new prospects for its clinical application in the treatment of hepatic diseases.

4.6. Other Biological Activities. Other biological effects include fibrinolytic, antipyretic, analgesic, antiatopic, antibacterial, anticancer/antitumor, and antidiabetic activities (Table 1). Two novel glucopyranosyl diacylglycerols (Figure 1(d)) isolated from $S$. fulvellum with a backbone composed of 1-O-palmitoyl-2-O-oleoyl-3-O-( $\alpha$-D-glucopyranosyl)-glycerol (POGG) and 1-O-myristoyl-2-O-oleoyl-3-O- $(\alpha$-D-glucopyranosyl)-glycerol (MOGG) were shown to exhibit fibrinolytic activity in a reaction system comprising pro-u-PA and plasminogen [27]. Kang et al. [11] revealed that tetradecanoic acid, hexadecanoic acid, neophytadiene, and oleic acid from S. fulvellum show potent antipyretic, analgesic, and antiinflammatory activities, especially antiedema activity. Yoon et al. [74] reported that the ethanol extract from S. fulvellum (SFE) showed antibacterial activity and that 70\% SFE significantly inhibited the $\alpha$-glucosidase activity in vitro, with an 
$\mathrm{IC}_{50}$ value of $8.13 \mu \mathrm{g} / \mathrm{mL}$ [75]. Another recent report revealed that the $\alpha$-glucosidase inhibitory activity can be enhanced using S. fulvellum enzymatic extracts, which also exhibited potent antioxidant and alcohol dehydrogenase activities [76]. Additionally, Jo et al. [77] reported the antitumoral effects of S. fulvellum hot-water extract and elucidated the potential mechanisms using both in vitro and in vivo systems. They found that the modulation of the Bcl-2 family members and activation of the p53 pathway may be closely related to the prevention of cancer and may therefore function as an important antitumoral mechanism.

\section{Conclusion and Future Perspectives}

S. fulvellum is a popular and inexpensive edible brown seaweed that is rich in nutrients (carbohydrates, unsaturated fatty acids, protein composition, vitamins, and minerals) and in natural bioactive compounds, such as phlorotannins, grasshopper ketones, and polysaccharides. Over the past twenty years, phytochemical investigations have led to the isolation of a large number of bioactive SFPs and other purified fractions. Bioactive low-molecule compounds (phlorotannins, fucoxanthin, astaxanthin, canthaxanthin, peridinin, fucoxanthinol, etc.) and polysaccharides are considered the major constituents of $S$. fulvellum that show many pharmacological effects, including antioxidant, immunomodulatory, anti-inflammatory, hepatoprotective, anticoagulant, and neuroprotective activities. However, systemic data are still insufficient regarding the pharmacokinetics and toxicity of this seaweed, especially target-organ toxicity; therefore, more investigations should be performed in the future. Furthermore, the biological activities of polysaccharides are closely related to their rheological properties, MW, chemical structures, constituent monosaccharides, substituent groups, and chain conformations [78]; thus, in-depth structural analysis of SFP needs to be systematically performed to assess the exact chemical structures of SFP.

More importantly, some issues still need to be taken into consideration; these include (1) the extraction method used for SFP preparation, which is currently exclusively hotwater extraction; (2) the lack of uniformity in raw materials and extraction and purification methods, implying SFP with differences in structure and biological activities; (3) the effects on human tissues that need to be studied, as in vitro cell experiments cannot completely reflect the complexity of a real organism-level response; (4) unclear bioactive components which are responsible for each bioactivity; (5) the fact that the development of the physiological activity of $S$. fulvellum extracts also presents its own limitations; therefore, future developments with respect to novel SFP extraction techniques (such as ultrasound, ultrahigh pressure, and multitechnology combination) are needed. Standard separation and purification methods also need to be established to obtain a homogeneous product, i.e., polysaccharides with high purity, high bioactivity, and high reproducibility. Moreover, in order to better determine the authenticity of $S$. fulvellum extracts on human health, in vivo experiments and clinical studies must be performed. Furthermore, in order to employ S. fulvellum for a wider range of applications, we recommend prompt screening and isolation to develop novel functional compounds from this seaweed.

\section{Additional Points}

Summary of the Literature. This review is aimed at presenting highlights from literature to provide novel insights into the potential of Sargassum fulvellum and its active compounds for use as innovative therapies. Section 1, which is Introduction, provides an overview of the general properties of $S$. fulvellum. Section 3 focuses on the key biologically active components of $S$. fulvellum, including the structure of $S$. fulvellum polysaccharides, their characterization, and composition; all these compounds have the potential to be developed as components of functional foods. Finally, Section 4 analyzes past studies on the pharmacological properties of S. fulvellum, which were divided into antioxidant, neuroprotective, immune-inflammatory, anticoagulative, and hepatoprotective; in each subsection, we focused on our increasing comprehension, highlighting the benefits of this macroalgae in human disease treatment. The present review discusses the phytochemical, pharmacological, therapeutic, nutritional, and health benefits of S. fulvellum, as well as its use in the prevention of diseases and maintenance of good health.

\section{Conflicts of Interest}

The authors report no conflicts of interest.

\section{Authors' Contributions}

Conceptualization was handled by J.L. and H.T.; resources were secured by J.L., S.L., and Q.W.; original draft preparation was taken care of by J.L.; review and editing were made by J.C. and H.T.; supervision was handled by M.W., H.T., and J.C.; and funding was acquired by M.W. and J.C.

\section{Acknowledgments}

We gratefully thank Dr. Alan K Chang (Wenzhou University) for critical reading of the manuscript. This work was financially supported by the National Natural Science Foundation of China (41876197), the National Key Research and Development Project (2018YFD0901503), the Natural Science Foundation of Zhejiang Province (LY18C020006), the Wenzhou Science and Technology Project (ZY2019013 and Y20180210), and the Golden Seed Project Grant from Korean Ministry of Oceans and Fisheries (213008-05-4-SB910).

\section{References}

[1] S. Gupta and N. Abu-Ghannam, "Bioactive potential and possible health effects of edible brown seaweeds," Trends in Food Science \& Technology, vol. 22, no. 6, pp. 315-326, 2011.

[2] A. R. Múzquiz de la Garza, M. Tapia-Salazar, M. MaldonadoMuñiz et al., "Nutraceutical potential of five Mexican brown seaweeds," BioMed Research International, vol. 2019, Article ID 3795160, 15 pages, 2019. 
[3] R. Peñalver, J. M. Lorenzo, G. Ros, R. Amarowicz, M. Pateiro, and G. Nieto, "Seaweeds as a functional ingredient for a healthy diet," Marine Drugs, vol. 18, no. 6, p. 301, 2020.

[4] A. Gomez-Zavaglia, M. A. Prieto Lage, C. Jimenez-Lopez, J. C. Mejuto, and J. Simal-Gandara, "The potential of seaweeds as a source of functional ingredients of prebiotic and antioxidant value," Antioxidants, vol. 8, no. 9, p. 406, 2019.

[5] B. H. Lee, B. W. Choi, J. H. Chun, and B. S. Yu, "Extraction of water soluble antioxidants from seaweeds," Applied Chemistry for Engineering, vol. 7, no. 6, pp. 1069-1077, 1996.

[6] M. J. Kim, S. M. Jeong, B. K. Kang, K. B. W. R. Kim, and D. H. Ahn, "Anti-inflammatory effects of grasshopper ketone from Sargassum fulvellum ethanol extract on lipopolysaccharideinduced inflammatory responses in RAW 264.7 cells," Journal of Microbiology and Biotechnology, vol. 29, no. 5, pp. 820-826, 2019.

[7] D.-S. Choi, Y. Athukorala, Y.-J. Jeon, M. Senevirathne, K.R. Cho, and S.-H. Kim, "Antioxidant activity of sulfated polysaccharides isolated from Sargassum fulvellum," Preventive Nutrition and Food Science, vol. 12, no. 2, pp. 65-73, 2007.

[8] E. K. Hwang, J. M. Baek, and C. S. Park, “Assessment of optimal depth and photon irradiance for cultivation of the brown alga, Sargassum fulvellum (Turner) C. Agardh," Journal of Applied Phycology, vol. 19, no. 6, pp. 787-793, 2007.

[9] K. K. A. Sanjeewa and Y. J. Jeon, "Edible brown seaweeds: a review," Journal of Food Bioactives, vol. 2, pp. 37-50, 2018.

[10] W. G. Gwon, M. S. Lee, J. S. Kim et al., "Hexane fraction from Sargassum fulvellum inhibits lipopolysaccharide-induced inducible nitric oxide synthase expression in RAW 264.7 cells via NF- $\kappa \mathrm{B}$ pathways," The American Journal of Chinese Medicine, vol. 41, no. 3, pp. 565-584, 2013.

[11] J. Y. Kang, M. N. A. Khan, N. H. Park et al., "Antipyretic, analgesic, and anti-inflammatory activities of the seaweed Sargassum fulvellum and Sargassum thunbergii in mice," Journal of Ethnopharmacology, vol. 116, no. 1, pp. 187190, 2008.

[12] M. A. Hannan, J. Y. Kang, Y. K. Hong et al., “A brown alga Sargassum fulvellum facilitates neuronal maturation and synaptogenesis," In Vitro Cellular \& Developmental Biology - Animal, vol. 48, no. 8, pp. 535-544, 2012.

[13] N. Y. Sung, H. M. Kim, E. B. Byun et al., "Polysaccharide extracted from Sargassum fulvellum leads to macrophage activation and Th1 polarization in splenocytes," Fisheries Science, vol. 81, no. 4, pp. 777-785, 2015.

[14] M. Fujihara, N. Iizima, I. Yamamoto, and T. Nagumo, "Purification and chemical and physical characterisation of an antitumour polysaccharide from the brown seaweed Sargassum fulvellum," Carbohydrate Research, vol. 125, no. 1, pp. 97106, 1984.

[15] M. De Zoysa, C. Nikapitiya, Y. J. Jeon, Y. Jee, and J. Lee, “Anticoagulant activity of sulfated polysaccharide isolated from fermented brown seaweed Sargassum fulvellum," Journal of Applied Phycology, vol. 20, no. 1, pp. 67-74, 2008.

[16] C. Barrow and F. Shahidi, Marine Nutraceuticals and Functional Foods, CRC Press, 2007.

[17] M. Kamiya, T. Nishio, A. Yokoyama et al., "Seasonal variation of phlorotannin in sargassacean species from the coast of the Sea of Japan," Phycological Research, vol. 58, no. 1, pp. 5361, 2010.

[18] N. M. Targett and T. M. Arnold, "Minireview-predicting the effects of brown algal phlorotannins on marine herbivores in tropical and temperate oceans," Journal of Phycology, vol. 34, no. 2, pp. 195-205, 1998.

[19] Y. X. Li, I. Wijesekara, Y. Li, and S. K. Kim, "Phlorotannins as bioactive agents from brown algae," Process Biochemistry, vol. 46, no. 12, pp. 2219-2224, 2011.

[20] T. I. Imbs and T. N. Zvyagintseva, "Phlorotannins are polyphenolic metabolites of brown algae," Russian Journal of Marine Biology, vol. 44, no. 4, pp. 263-273, 2018.

[21] R. Zhang, A. K. L. Yuen, M. Magnusson et al., "A comparative assessment of the activity and structure of phlorotannins from the brown seaweed Carpophyllum flexuosum," Algal Research, vol. 29, pp. 130-141, 2018.

[22] Y. Kamei and C. K. Tsang, "Sargaquinoic acid promotes neurite outgrowth via protein kinase A and MAP kinases-mediated signaling pathways in PC12D cells," International Journal of Developmental Neuroscience, vol. 21, no. 5, pp. 255-262, 2003.

[23] T. Matsuno, "Aquatic animal carotenoids," Fisheries Science, vol. 67, no. 5, pp. 771-783, 2001.

[24] J. Peng, J. P. Yuan, C. F. Wu, and J. H. Wang, "Fucoxanthin, a marine carotenoid present in brown seaweeds and diatoms: metabolism and bioactivities relevant to human health," Marine Drugs, vol. 9, no. 10, pp. 1806-1828, 2011.

[25] K. Miyashita and M. Hosokawa, "Health impact of marine carotenoids," Journal of Food Bioactives, vol. 1, pp. 31-40, 2018.

[26] A. Ina, K. I. Hayashi, H. Nozaki, and Y. Kamei, "Pheophytin a, a low molecular weight compound found in the marine brown alga Sargassum fulvellum, promotes the differentiation of PC12 cells," International Journal of Developmental Neuroscience, vol. 25, no. 1, pp. 63-68, 2007.

[27] W. Wu, K. Hasumi, H. Peng, X. Hu, X. Wang, and B. Bao, "Fibrinolytic compounds isolated from a brown alga, Sargassum fulvellum," Marine Drugs, vol. 7, no. 2, pp. 8594, 2009.

[28] A. S. Marriott, A. J. Hunt, E. Bergström, J. Thomas-Oates, and J. H. Clark, "Effect of rate of pyrolysis on the textural properties of naturally-templated porous carbons from alginic acid," Journal of Analytical and Applied Pyrolysis, vol. 121, pp. 62-66, 2016.

[29] L. Wang, Y. J. Park, Y. J. Jeon, and B. M. Ryu, "Bioactivities of the edible brown seaweed, Undaria pinnatifida: a review," Aquaculture, vol. 495, pp. 873-880, 2018.

[30] S. Luthuli, S. Y. Wu, Y. Cheng, X. L. Zheng, M. J. Wu, and H. B. Tong, "Therapeutic effects of fucoidan: a review on recent studies," Marine Drugs, vol. 17, no. 9, pp. 487-502, 2019.

[31] K. K. A. Sanjeewa, J. S. Lee, W. S. Kim, and Y. J. Jeon, “The potential of brown-algae polysaccharides for the development of anticancer agents: an update on anticancer effects reported for fucoidan and laminaran," Carbohydrate Polymers, vol. 177, pp. 451-459, 2017.

[32] M. Plaza, A. Cifuentes, and E. Ibáñez, "In the search of new functional food ingredients from algae," Trends in Food Science and Technology, vol. 19, no. 1, pp. 31-39, 2008.

[33] A. J. Smit, "Medicinal and pharmaceutical uses of seaweed natural products: a review," Journal of Applied Phycology, vol. 16, no. 4, pp. 245-262, 2004.

[34] J. H. Jo, D. Kim, S. Lee, and T. K. Lee, "Total phenolic contents and biological activities of Korean seaweed extracts," Food Science and Biotechnology, vol. 14, no. 6, pp. 1-5, 2005.

[35] J. Liu, R. Y. Bai, Y. P. Liu, X. Zhang, J. Kan, and C. H. Jin, "Isolation, structural characterization and bioactivities of naturally 
occurring polysaccharide-polyphenolic conjugates from medicinal plants-a reivew," International Journal of Biological Macromolecules, vol. 107, no. Part B, pp. 2242-2250, 2018.

[36] L. Wang, Y. J. Park, J. Y. Oh, and I. P. S. Fernando, "Protective effects of enzyme-assistant extracts of Sargassum fulvellum against AAPH-induced oxidative stress in vitro in Vero cells," Journal of Chitin and Chitosan, vol. 23, no. 2, pp. 113-119, 2018.

[37] S. J. Heo, S. H. Cha, K. W. Lee, S. M. K. Cho, and Y. J. Jeon, "Antioxidant activities of chlorophyta and phaeophyta from Jeju Island," Algae, vol. 20, no. 3, pp. 251-260, 2005.

[38] J. W. Wu, P. Li, D. B. Tao et al., "Effect of solution plasma process with hydrogen peroxide on the degradation and antioxidant activity of polysaccharide from Auricularia auricula," International Journal of Biological Macromolecules, vol. 117, pp. 1299-1304, 2018.

[39] F. M. Ma, J. W. Wu, P. Li et al., "Effect of solution plasma process with hydrogen peroxide on the degradation of watersoluble polysaccharide from Auricularia auricula. II: solution conformation and antioxidant activities in vitro," Carbohydrate Polymers, vol. 198, pp. 575-580, 2018.

[40] Y. X. Sun, J. C. Liu, and J. F. Kennedy, "Purification, composition analysis and antioxidant activity of different polysaccharide conjugates (APPs) from the fruiting bodies of Auricularia polytricha," Carbohydrate Polymers, vol. 82, no. 2, pp. 299-304, 2010.

[41] T. K. Chaudhuri and S. Paul, "Protein-misfolding diseases and chaperone-based therapeutic approaches," The FEBS Journal, vol. 273, no. 7, pp. 1331-1349, 2006.

[42] F. Raynaud and A. Marcilhac, "Implication of calpain in neuronal apoptosis," The FEBS Journal, vol. 273, no. 15, pp. 3437-3443, 2006.

[43] Y. Sari, "Huntington's disease: from mutant huntingtin protein to neurotrophic factor therapy," International Journal of Biomedical Sciences, vol. 7, no. 2, pp. 89-100, 2011.

[44] D. Z. Christensen, T. A. Bayer, and O. Wirths, "Intracellular $\mathrm{A} \beta$ triggers neuron loss in the cholinergic system of the APP/PS1KI mouse model of Alzheimer's disease," Neurobiology of Aging, vol. 31, no. 7, pp. 1153-1163, 2010.

[45] M. A. Hannan, J. Y. Kang, Y. K. Hong et al., "The marine alga Gelidium amansiipromotes the development and complexity of neuronal cytoarchitecture," Phytotherapy Research, vol. 27, no. 1, pp. 21-29, 2013.

[46] C. K. Tsang, A. Ina, T. Goto, and Y. Kamei, "Sargachromenol, a novel nerve growth factor-potentiating substance isolated from Sargassum macrocarpum, promotes neurite outgrowth and survival via distinct signaling pathways in PC12D cells," Neuroscience, vol. 132, no. 3, pp. 633-643, 2005.

[47] A. Ina and Y. Kamei, "Vitamin B(12), a chlorophyll-related analog to pheophytin a from marine brown algae, promotes neurite outgrowth and stimulates differentiation in $\mathrm{PC} 12$ cells," Cytotechnology, vol. 52, no. 3, pp. 181-187, 2006.

[48] E. J. Shin, E. J. Han, M. J. Kim, J. Jung, J. Park, and G. Ahn, "Inhibition effect of a hot water extracts from Sargassum fulvellum againstgluten-induced inflammation and oxidative damage in human keratinocytes," Journal of Chitin and Chitosan, vol. 24, no. 2, pp. 90-98, 2019.

[49] A. Cumashi, N. A. Ushakova, M. E. Preobrazhenskaya et al., "A comparative study of the anti-inflammatory, anticoagulant, antiangiogenic, and antiadhesive activities of nine different fucoidans from brown seaweeds," Glycobiology, vol. 17, no. 5, pp. 541-552, 2007.
[50] S. M. Kang, K. N. Kim, S. H. Lee et al., "Anti-inflammatory activity of polysaccharide purified from AMG-assistant extract of Ecklonia cava in LPS-stimulated RAW 264.7 macrophages," Carbohydrate Polymers, vol. 85, no. 1, pp. 80-85, 2011.

[51] J. Tang, H. Yan, and S. Zhuang, "Inflammation and oxidative stress in obesity-related glomerulopathy," International Journal of Nephrology, vol. 2012, Article ID 608397, 2012.

[52] W. Wijesinghe and Y. J. Jeon, "Enzyme-assistant extraction (EAE) of bioactive components: a useful approach for recovery of industrially important metabolites from seaweeds: a review," Fitoterapia, vol. 83, no. 1, pp. 6-12, 2012.

[53] H. A. Monsur, I. Jaswir, S. Simsek, A. Amid, Z. Alam, and A. H. Tawakalit, "Cytotoxicity and inhibition of nitric oxide syntheses in LPS induced macrophage by water soluble fractions of brown seaweed," Food Hydrocolloids, vol. 42, Part 2, pp. 269-274, 2014.

[54] E. H. Byun, "Comparison study of immunomodulatory activity of polysaccharide and ethanol extracted from Sargassum fulvellum," Journal of the Korean Society of Food Science and Nutrition, vol. 44, no. 11, pp. 1621-1628, 2015.

[55] D. H. Jeong, K. B. W. R. Kim, B. K. Kang et al., "Anti-inflammatory activity of the water extract of Sargassum fulvellum," Korean Society for Biotechnology and Bioengineering Journal, vol. 27, no. 6, pp. 325-329, 2012.

[56] I. Jaswir, H. A. Monsur, S. Simsek et al., "Cytotoxicity and inhibition of nitric oxide in lipopolysaccharide induced mammalian cell lines by aqueous extracts of brown seaweed," Journal of Oleo Science, vol. 63, no. 8, pp. 787-794, 2014.

[57] M. J. Kim, K. B. W. R. Kim, D. H. Jeong, and D. H. Ahn, "Inhibitory effect of Sargauum fulvellum ethanolic extract on LPS-induced inflammatory reaction in RAW 264.7 mouse macrophages," Journal of Applied Biological Chemistry, vol. 56, no. 4, pp. 249-255, 2013.

[58] B. K. Kang, M. J. Kim, K. B. W. R. Kim, and D. H. Ahn, "In vivo and in vitro inhibitory activity of an ethanolic extract of Sargassum fulvellum and its component grasshopper ketone on atopic dermatitis," International Immunopharmacology, vol. 40, pp. 176-183, 2016.

[59] J. A. Nichols and S. K. Katiyar, "Skin photoprotection by natural polyphenols: anti-inflammatory, antioxidant and DNA repair mechanisms," Archives of Dermatological Research, vol. 302, no. 2, pp. 71-83, 2010.

[60] J. K. Kim, Y. Kim, K. M. Na, Y. J. Surh, and T. Y. Kim, “[6]Gingerol prevents UVB-induced ROS production and COX2 expression in vitro and in vivo," Free Radical Research, vol. 41, no. 5, pp. 603-614, 2007.

[61] A. Nicolaou, S. M. Pilkington, and L. E. Rhodes, "Ultravioletradiation induced skin inflammation: dissecting the role of bioactive lipids," Chemistry and Physics of Lipids, vol. 164, no. 6, pp. 535-543, 2011.

[62] C. Lee, G. H. Park, E. M. Ahn, C. I. Park, and J. H. Jang, "Sargassum fulvellum protects $\mathrm{HaCaT}$ cells and $\mathrm{BALB} / \mathrm{c}$ mice from UVB-induced proinflammatory responses," Evidence-Based Complementary and Alternative Medicine, vol. 2013, Article ID 747846, 10 pages, 2013.

[63] C. Faggio, M. Pagano, A. Dottore, G. Genovese, and M. Morabito, "Evaluation of anticoagulant activity of two algal polysaccharides," Natural Product Research, vol. 30, no. 17, pp. 1934-1937, 2016.

[64] U. R. Desai, "New antithrombin-based anticoagulants," Medicinal Research Reviews, vol. 24, no. 2, pp. 151-181, 2004. 
[65] I. Wijesekara, R. Pangestuti, and S. K. Kim, "Biological activities and potential health benefits of sulfated polysaccharides derived from marine algae," Carbohydrate Polymers, vol. 84, no. 1, pp. 14-21, 2011.

[66] Y. Athukorala, K. W. Lee, S. K. Kim, and Y. J. Jeon, “Anticoagulant activity of marine green and brown algae collected from Jeju Island in Korea," Bioresource Technology, vol. 98, no. 9, pp. 1711-1716, 2007.

[67] K. Matsubara, "Recent advances in marine algal anticoagulants," Current Medicinal Chemistry-Cardiovascular \& Hematological Agents, vol. 2, no. 1, pp. 13-19, 2004.

[68] Y. Athukorala, W. K. Jung, T. Vasanthan, and Y. J. Jeon, “An anticoagulative polysaccharide from an enzymatic hydrolysate of Ecklonia cava," Carbohydrate Polymers, vol. 66, no. 2, pp. 184-191, 2006.

[69] B. W. Jo and S. K. Choi, "Degradation of fucoidans from Sargassum fulvellum and their biological activities," Carbohydrate Polymers, vol. 111, pp. 822-829, 2014.

[70] E. Madrigal Santillán, E. Madrigal Bujaidar, I. Álvarez González et al., "Review of natural products with hepatoprotective effects," World Journal of Gastroenterology, vol. 20, no. 40, pp. 14787-14804, 2014.

[71] N. Mori, K. Nakasone, K. Tomimori, and C. Ishikawa, "Beneficial effects of fucoidan in patients with chronic hepatitis $\mathrm{C}$ virus infection," World Journal of Gastroenterology, vol. 18, no. 18, pp. 2225-2230, 2012.

[72] N. Kawano, Y. Egashira, and H. Sanada, "Effect of dietary fiber in edible seaweeds on the development of D-galactosamineinduced hepatopathy in rats," Journal of Nutritional Science and Vitaminology, vol. 53, no. 5, pp. 446-450, 2007.

[73] N. Kawano, Y. Egashira, and H. Sanada, "Effects of various kinds of edible seaweeds in diets on the development of Dgalactosamine-induced hepatopathy in rats," Journal of Nutritional Science and Vitaminology, vol. 53, no. 4, pp. 315-323, 2007.

[74] S. Y. Yoon, S. Y. Lee, K. B. W. R. Kim et al., “Antimicrobial activity of the Sargassum fulvellum ethanol extract and the effect of temperature and $\mathrm{pH}$ on their activity," Korean Journal of Food Science and Technology, vol. 42, no. 2, pp. 155-159, 2010.

[75] S. Y. Jeong, Z. J. Qian, Y. J. Jin, G. O. Kim, P. Y. Yun, and T. O. Cho, "Investigation of $\alpha$-giucosidase inhibitory activity of ethanolic extracts from 19 species of marine macroalgae in Korea," Natural Product Sciences, vol. 18, no. 2, pp. 130-136, 2012.

[76] E. K. Cho, S. H. Kang, and Y. J. Choi, "Biological analysis of enzymatic extracts from Sargassum fulvellum using polysaccharide degrading enzyme," Korean Society for Biotechnology and Bioengineering Journal, vol. 28, no. 6, pp. 349-355, 2013.

[77] E. H. Jo, S. D. Cho, N. S. Ahn et al., "Inhibition of human breast carcinoma by BLC (Sargassum fulvellum) and BLC/HEN egg in vitro and in vivo," Korean Journal of Veterinary Research, vol. 45, pp. 85-91, 2005.

[78] S. P. Wasser, "Medicinal mushrooms as a source of antitumor and immunomodulating polysaccharides," Applied Microbiology and Biotechnology, vol. 60, no. 3, pp. 258-274, 2002.

[79] D. H. Jeong, K. B. W. R. Kim, M. J. Kim et al., “Anti-inflammatory effect of ethanol extract from Sargassum fulvellum on lipopolysaccharide induced inflammatory responses in RAW 264.7 cells and mice ears," Journal of the Korean Society of Food Science and Nutrition, vol. 43, no. 8, pp. 1158-1165, 2014.
[80] J. H. Oh, J. Kim, and Y. Lee, "Anti-inflammatory and antidiabetic effects of brown seaweeds in high-fat diet-induced obese mice," Nutrition Research and Practice, vol. 10, no. 1, pp. 42-48, 2016.

[81] D. H. Jeong, N. K. Ahn, Y. U. Choi et al., "Inhibitory effect of Sargassum fulvellum water extract on 2,4-dinitrochlorobenzene-induced atopic dermatitis-like skin lesions in mice," Microbiology and Biotechnology Letters, vol. 43, no. 2, pp. 150-157, 2015.

[82] M. Fujihara, K. Komiyama, I. Umezawa, and T. Nagumo, "Antitumor activity and its mechanisms of sodium alginate from the brown seaweed Sargassum fulvellum," Chemotherapy, vol. 32, no. 12, pp. 1004-1009, 1984.

[83] I. Yamamoto, T. Nagumo, M. Fujihara, M. Takahashi, and Y. Ando, "Antitumor effect of seaweeds. II. Fractionation and partial characterization of the polysaccharide with antitumor activity from Sargassum fulvellum," The Japanese Journal of Experimental Medicine, vol. 47, no. 3, pp. 133-140, 1977. 